\title{
DESENVOLVIMENTO DE UM PORTAL PARA REPRESENTAÇÕES DE VARIÁVEIS RELACIONADAS A PERTURBAÇÕES GEOMAGNÉTICAS
}

\author{
Rodrigo Takeshi Seo' \\ José Paulo Marchezi ${ }^{2}$ \\ Emanuel Mineda Carneiro ${ }^{3}$ \\ Odim Mendes ${ }^{4}$ \\ Margarete Oliveira Domingues ${ }^{5}$
}

Resumo: Perturbações geomagnéticas podem ser classificadas por meio de índice, como o Disturbance storm-time index (Dst). Este trabalho detalha o processo de desenvolvimento de um sistema computacional que visa apresentar aos usuários representações gráficas das variações geomagnéticas geradas no ambiente solar-terrestre. Foi criada, de forma programática, uma rotina de obtenção de dados geomagnéticos de diversos observatórios, disponibilizados pela International Real-time Magnetic Observatory Network (INTERMAGNET) e armazenados em uma base de dados localizada no Instituto Nacional de Pesquisas Espaciais e utilizados para a obtenção dos índices geomagnéticos, dentre eles, uma variação do cálculo do índice Dst, utilizando técnicas baseadas em Wavelet (WISA-Dst) e as variações das componentes do campo geomagnético HDZ.

Palavras-chave: Desenvolvimento de software; Aplicação web; Banco de dados; Geomagnetismo; Clima espacial.

\footnotetext{
1 Divisão de Geofísica Espacial (DGE), Instituto Nacional de Pesquisas Espaciais, Faculdade de Tecnologia de São José dos Campos - Professor Jessen Vidal, Brasil. E-mail: rodrigo.seo@inpe.br.

2 Divisão de Geofísica Espacial (DGE), Instituto Nacional de Pesquisas Espaciais, Brasil. E-mail: jose.marchezi@inpe.br.

${ }^{3}$ Faculdade de Tecnologia de São José dos Campos - Professor Jessen Vidal, Brasil. E-mail: emanuel.mineda@fatec.sp.gov.br.

4 Divisão de Geofísica Espacial (DGE), Instituto Nacional de Pesquisas Espaciais, Brasil. E-mail: odim.mendes@inpe.br.

5 Laboratório Associado de Computação e Matemática Aplicada (LAC), Instituto Nacional de Pesquisas Espaciais, Brasil. E-mail: margarete.domingues@inpe.br.
} 